\title{
Culture in vitro de la renoncule des fleuristes (Ranunculus asiaticus L). III Étude des plantes produites par embryogenèse somatique à partir des tissus superficiels de l'anthère
}

\author{
J Meynet *, A Duclos \\ INRA, station d'amélioration des plantes florales, la Gaudine, Fréjus, 83370 Saint-Aygulf, France
}

(Reçu le 5 septembre 1989 ; accepté le 29 janvier 1990)

\begin{abstract}
Résumé - Les tissus superficiels de l'anthère de $R$ asiaticus peuvent donner par embryogenèse directe, sans cal apparent, des plantes vigoureuses, généralement diploïdes, rarement tétraploïdes ou aneuploïdes, jamais haploïdes, de même structure allélique $S$ (sauf mutation) que leur donneur. Malgré leur origine somatique, ces plantes présentent une variation somaclonale extrêmement importante qui affecte, notamment, la couleur des fleurs pour 2 donneurs bicolores. Une plante s'est révélée autofertile. Les caractères nouveaux sont stables après 1 année de culture en serre ou de multiplication in vitro, ils se transmettent dans les descendances sexuées par la voie mâle.
\end{abstract}

Ranunculus asiaticus / anthère / culture in vitro / variation somaclonale / embryogenèse

\begin{abstract}
Summary - In vitro culture of Persian buttercup (Ranunculus asiaticus L). III. Study of plants regenerated by somatic embryogenesis from the anther superficial tissues. Plants without apparent callus can be obtained by direct embryogenesis from the superficial tissues of anthers in $\mathrm{R}$ asiaticus. The plants are vigourous, generally diploid, rarely tetraploid or aneuploid, never haploid. They have the same allelic $S$ structure as their donor (except for mutation). In spite of their somatic origin, these plants present a very important somaclonal variation which especially concerns the flower colour for 2 bicoloured donors (table I). In particular among the 17 somatic issues from a yellow with red fringe genotype, 10 have a homogeneous red colour and 7 remain bicoloured but the anthocyanic border of their petals is fairly wide. One plant appears self-compatible and cross-compatible with the 5 plants tested and used as females which were of the same donor origin (table II). The new characters are stable after cultivation under greenhouse conditions and in vitro multiplication for 1 year. Nine crosses between different plants obtained through somatic embryogenesis and an acyanic test plant indicate a very good transmissibility of the new specificities (colour and selfcompatibility) from 1 generation to the next (table III).
\end{abstract}

Ranunculus asiaticus / anther culture / in vitro culture / somaclonal variation / embryogenesis

\section{INTRODUCTION}

La culture in vitro d'anthères de renoncule a permis d'obtenir 2 types d'explants selon le génotype du donneur:

- des embryons «Em» adhérents à l'épiderme de l'anthère indéhiscente et évoluant directement en plantes,

- des cals ou nodules «C" provenant de l'intérieur du sac pollinique et capables de régénérer des plantes viables (Meynet et Duclos, 1990b). L'étude présentée ici ne porte que sur les plants Em. A défaut d'observations histologiques décisives, nous nous attacherons dans un premier temps à démontrer l'origine somatique de ces plantes $\mathrm{Em}$, puis nous analyserons la variation phénotypique observée. Cette étude nécessite la connaissance d'un certain nombre de marqueurs génétiques stables et mesurables qui ont fait l'objet de travaux antérieurs (Meynet, 1974) et que nous rappelons ici sommairement.

- La richesse des coloris (peu dépendants du milieu environnant) exprime une grande diversité d'équilibres pigmentaires. La présence d'anthocyane est contrôlée par un gène dominant $A$, les types acyaniques étant récessifs aa, par ailleurs la présence de delphinidine (généralement associée à la cyanidine) est monogénique (Dp) et dominante par rapport à l'absence. En revanche, les concentrations et répartitions anthocyaniques sont déterminées par une hérédité polygénique compliquée.

" Correspondance et tirés à part 
- l'auto-incompatibilité gamétophytique contrôlée par une seule série allélique $S$ est de règle chez cette espèce. Cependant ce caractère est parfois imparfait et fluctuant : dans nos conditions expérimentales, quelques graines (en général moins de 50 , à comparer à plusieurs centaines possibles après croisement) peuvent être obtenues après autofécondation, en mars-avril lorsque le temps est frais et couvert, alors qu'aux Pays-Bas et en Angleterre la plupart des renoncules se révèleraient généralement autofertiles (Godart, communication personnelle).

- les effets de la consanguinité sont très nets sur les dimensions de différents organes végétatifs ou floraux, le nombre de feuilles de la rosette, la duplicature, la production grainière, mais ils affectent peu la précocité de floraison.

\section{MATÉRIEL ET MÉTHODES}

\section{Les génotypes donneurs}

\section{La plante H3059.7}

Elle est issue d'une population hybride Friandine caractérisée en particulier par des fleurs bicolores : sur un fond homogène jaune (caroténoïdes) les pétales sont marginés d'une étroite plage anthocyanée. L'anthocyane présente est la cyanidine et la plante est homozygote AA, dpdp. De ce génotype nous avons obtenu 17 plantes Em qui ont fleuri dès le printemps 1987.

Deux plantes dérivées de $\mathrm{H} 3059.7$ : $\mathrm{A} 1$ et $\mathrm{A} 4$ ont été réutilisées pour un $2^{\mathrm{e}}$ cycle d'embryogenèse.

\section{Le clone parental \$7002.6}

Ses pétales présentent un liseré anthocyané (delphinidine) sur fond jaune pâle. Cette plante est hétérozygote Aa, Dpdp; 21 plantes Em ont fleuri en 1989.

\section{Le clone $\mathbf{S 4 0 3 2 . 2 3}$}

II produit de grosses fleurs orange très doubles; 2 plantes Em issues d'individus de ce clone ont fleuri en 1989.

\section{Le clone S5145.24}

Il présente des fleurs vermillon; la face inférieure des feuilles juvéniles est fortement anthocyanée et les in- florescences sont relativement courtes; 3 plantes Em issues de ce clone ont fleuri en 1989.

\section{La production des embryons in vitro}

La technique de culture in vitro, déjà décrite dans un précédent article (Meynet et Duclos, 1990b), est simplement résumée ici.

Les anthères sont prélevées en mars sur le $1^{\mathrm{er}}$ bouton axillaire alors que les sépales du bouton terminal s'entrouvent et laissent apparaître la couleur. Elles sont disposées sur un milieu gélosé contenant notamment des macroéléments de Murashige et Skoog dilués de moitié et $1 \mathrm{mg} . \mathrm{I}^{-1}$ de benzyladénine. Les cultures sont placées pendant $14 \mathrm{j}$ à $27^{\circ} \mathrm{C}$ et à l'obscurité, puis demeurent dans une chambre éclairée pendant $12 \mathrm{~h}$ à $18^{\circ} \mathrm{C}$ jusqu'à l'émergence des embryons. Ceux-ci sont transplantés sur un milieu de prolifération où ils se développent directement en plantules. On obtient ainsi pour chaque embryon 2 à 6 plantes qui sont observées dans une serre en verre dont la température minimale est de $4^{\circ} \mathrm{C}$.

\section{Les notations}

Les phénotypes, en particulier les caractères floraux, des plantes obtenues sont observés sur des plantes sorties de tubes; pour $\mathrm{H}$ 3059-7, nous avons pu renouveler ces notations l'année suivante sur des plantes provenant de griffes produites par les vitroplants ou issues de matériel entretenu in vitro pendant 1 année entière après 8 repiquages successifs.

Les coloris sont notés d'après des critères visuels (ton - répartition anthocyanique); la nature des anthocyanidines est parfois déterminée par chromatographie sur couche mince de cellulose; la concentration moyenne en anthocyane est mesurée par densité optique. Les pigments contenus dans 15 pétales (représentant un poids frais moyen de l'ordre de $1 \mathrm{~g}$ ) sont alors extraits dans $50 \mathrm{ml}$ d'éthanol avec $0,2 \%$ d'HCl; après broyage et filtration, la densité optique est déterminée à $525 \mathrm{~nm}$.

Des comptages chromosomiques sont réalisés sur pointes de racines, selon la technique de Feulgen.

Par ailleurs, les 17 plantes dérivées de $\mathrm{H} 3059-7$ ont été systématiquement autopollinisées (elles produisent toutes beaucoup de pollen); 3 d'entre elles servent de parents mâles dans un programme d'intercroisements à l'intérieur de cette population $\mathrm{H} 3059.7$ (A1 A4 - A15), chaque famille issue d'autofécondation sera représentée par 40 plantes en serre. Enfin 9 de ces plantes ont été utilisées comme parents mâles dans un programme de croisements avec un testeur commun S4032-1 acyanique (jaune pur), mâle-stérile et non apparenté à H3059.7. Chaque famille hybride sera représentée par 72 plantes. 


\section{RÉSULTATS}

\section{Comptage chromosomique}

Au total, 25 plantes ont fait l'objet d'un comptage chromosomique :

-22 sont diploïdes $(2 n=16)$,

-1 est trisomique $(2 n+1=17)$, elle a un faciès floral particulier qui suggère une structure en chimère, elle produit néanmoins du pollen normal et fonctionnel.

-1 est tétraploïde

-1 est mixoploïde (32/16), mais le doublement chromosomique de ces 2 dernières plantes n'affecte probablement que le système racinaire car elles ne se distinguent pas phénotypiquement de leurs voisins diploïdes, de plus, elles restent autostériles et leur descendance en croisement est normale.

\section{Variations observées}

\section{Synthèse anthocyanique}

Le tableau I montre que sur 17 plantes dérivées de $\mathrm{H} 3059.7,10$ présentent des pétales de couleur plus ou moins homogène et 7 restent bicolores, mais la taille du secteur anthocyané varie comme l'indiquent les valeurs de densité optique. En fait, tous les dérivés embryogénétiques apparaissent distincts de leur donneur et différents entre eux.

Cette observation se confirme chez les plantes dérivées de $\$ 7002.6$ : sur les 21 plantes observées, 11 ont des fleurs homogènes pourpres, 3 pourpres striées, 2 jaunes sans anthocyane apparente et 5 bicolores; parmi ces dernières 1 plante est à cyanidine seule, contrairement à toutes les autres plantes anthocyanées qui possèdent de la delphinidine.

En revanche les dérivés de $\$ 4032-23$ et S5145-24 à fleur de couleur homogène sont identiques à leur donneur respectif pour ce caractère.

Par ailleurs la confrontation des phénotypes observés sur le matériel H3059-7 (élevé à partir de griffes ou après une année de propagation in vitro) avec des photographies couleur prises l'année précédente sur des vitroplants révèle une grande stabilité phénotypique. De même, nous n'avons jamais observé de variation à l'intérieur des petits clones issus d'embryons isolés.

\section{Autofertilité}

La plante A15 dérivée de H3059-7 produit autant de graines en autofécondation (ou lors-

Tableau I. Quelques caractéristiques de $\mathrm{H} 3059.7$ et de ses 17 dérivés embryogéniques.

\begin{tabular}{|c|c|c|c|c|}
\hline & Couleur & $D O_{525}$ & Nbre chromosomique & Nbre anthères/fleur \\
\hline H3059.7 & jaune + orange & 0,350 & & 50 à 70 \\
\hline$A 1$ & orange + rouge & 0,507 & 16 & 50 à 70 \\
\hline $\mathrm{A} 2$ & jaune + rouge & 0,331 & 16 & 50 à 70 \\
\hline A3 & rouge orange strié & & & 30 à 50 \\
\hline A4 & rouge vermillon & 1,378 & 16 & 50 à 70 \\
\hline A5 & rouge orange & 0,585 & 16 & 50 à 70 \\
\hline A6 & rouge orange strié & & 16 & 50 à 70 \\
\hline$A 7$ & rouge striures foncées & & 17 & 30 à 50 \\
\hline A8 & jaune + rouge & & & 30 à 50 \\
\hline A9 & jaune + liseré rouge & & 16 & 50 à 70 \\
\hline A10 & jaune orange + rouge & 0,862 & $32 / 16$ & 50 à 70 \\
\hline A11 & jaune + rouge & 0,306 & & 50 à 70 \\
\hline A12 & rouge orange & & 16 & 50 à 70 \\
\hline$A 13$ & jaune + rouge & & & 50 à 70 \\
\hline A14 & orange + rouge & 0,366 & 16 & 50 à 70 \\
\hline A15 & rouge & 1,040 & 16 & 50 à 70 \\
\hline$A 16$ & rouge foncé & 1,520 & & 50 à 70 \\
\hline A17 & rouge & & & 30 à 50 \\
\hline
\end{tabular}


qu'elle est utilisée comme pollinisatrice de ses homologues dérivées du même $\mathrm{H}$ 3059-7) qu'en intercroisement par une plante non apparentée (tableau II). A15 est diploïde et elle est la seule plante devenue autofertile parmi celles testées.

\section{Autres caractères}

De nombreux autres caractères paraissent modifiés dans toutes les familles observées.

Ainsi la duplication varie sensiblement : 5 plantes dérivées de S7002-6, 1 de S4032-23 et 2 de S5145-24 sont restées entièrement doubles c'est-à-dire mâle-stériles.

Une plante S7002-6 bicolore présente des sépales très allongés.

Une dérivée de $\mathbf{5} 5145-23$ a des feuilles juvéniles relativement peu anthocyanées.

Les graines récoltées sur 1 clone provenant de S5145-23, malgré leur apparence normale, manifestent une dormance inhabituelle et n'ont pas encore pu germer.

\section{Autofécondation et croisements consanguins par A15}

Ces descendances présentent une dépression de vigueur uniforme et qui se traduit par un vo- lume foliaire, une taille et un nombre de fleurs réduits, une stérilité mâle très fréquente. Seules 5 plantes ont produit du pollen en fin de cycle, 3 d'entre elles se sont révélées autofertiles.

Ces familles montrent par ailleurs une très grande diversité phénotypique, traduisant une très forte hétérozygotie des parents.

\section{Hybridations par S4032-1}

Les hybrides retrouvent une grande vigueur, comparable à celle des dérivés de H3059-7. Les coloris plus ou moins hétérogènes sont distribués de façon continue. La concentration anthocyanique est déterminée par mesure de densité optique, chaque hybride est représenté par un échantillon moyen (chaque individu hybride fournissant 3 pétales). La très forte corrélation établie entre les 9 dérivés embryogéniques de H3059-7 et leurs hybrides respectifs par un testeur commun ( $r=0,945$ - tableau III) indique une bonne transmission génétique des caractères nouveaux par la voie mâle.

L'hybride S4032-1 x A15 a fait, par ailleurs, l'objet d'une analyse concernant l'hérédité de l'autofertilité. Trente-neuf plantes ont pu être autofécondées, les autres étant mâle-stériles comme leur parent femelle, 17 plantes sont autofertiles comme A15. L'hypothèse d'un détermi-

Tableau II. Nombre de graines produites après autopollinisation (AF), croisements entre dérivés de H 3059.7, hybridation par S4032.1.

\begin{tabular}{|c|c|c|c|c|c|}
\hline \multirow[b]{2}{*}{ Parent issu de H 3059.7} & \multicolumn{5}{|c|}{ Autre parent } \\
\hline & $A F$ & $A 4\left(0^{\prime \prime}\right)$ & $A 11\left(0^{\circ}\right)$ & $A 15\left(0^{\prime \prime}\right)$ & $S 4032.1(9)$ \\
\hline A1 & 15 & & & & 490 \\
\hline A2 & $26 ; 0$ & 0 & 0 & 284 & $348 ; 433$ \\
\hline A3 & 0 & 2 & 28 & & \\
\hline A4 & 0 & 0 & $8 ; 0$ & & 531 \\
\hline A5 & $0 ; 0$ & 42 & 0 & 495 & 405 \\
\hline A6 & $0 ; 0$ & 10 & 0 & & \\
\hline A7 & $0 ; 8$ & & 10 & & \\
\hline A8 & $12 ; 2$ & 0 & 0 & 293 & \\
\hline A9 & 0 & & & & \\
\hline A10 & & & & & 430 \\
\hline A11 & $0 ; 0$ & 19 & & $327 ; 410$ & 326 \\
\hline A12 & $0 ; 4$ & & & & \\
\hline A13 & 0 & & & & \\
\hline A14 & 0 & & 0 & & 324 \\
\hline A15 & $343 ; 314 ; 380$ & & & & $290 ; 387$ \\
\hline A16 & 0 & & & 370 & 395 \\
\hline A17 & $0 ; 39$ & & & & \\
\hline
\end{tabular}


Tableau III. Valeurs des mesures de densité optique à $525 \mathrm{~nm}$ d'extraits alcooliques de pétales de 9 dérivés embryogénétiques $\mathrm{H} 3059.7$ et de leurs hybrides relatifs par le testeur $S 4032.1$.

Embryons $\quad D O$ (plantes Em) DO (4032.1 $\times E m)$

\begin{tabular}{lll}
\hline A11 & 0,306 & 0,366 \\
A2 & 0,331 & 0,404 \\
A14 & 0,366 & 0,862 \\
A1 & 0,507 & 0,860 \\
A5 & 0,586 & 1,749 \\
A10 & 0,862 & 1,552 \\
A15 & 1,040 & 1,928 \\
A4 & 1,378 & 2,701 \\
A16 & 1,520 & 2,550 \\
\hline
\end{tabular}

nisme monofactoriel (pollen $S^{\star}$ capable de germer sur tout stigmate $S \times S^{*}$ ) rend compte de cette ségrégation $1 / 1\left(\chi^{2}=0,64\right)$.

\section{Deuxième cycle d'embryogenèse}

La plante $\mathrm{A} 1$ bicolore $\left(\mathrm{DO}_{525}=0,507\right)$ donne 2 dérivés bicolores et 7 rouge foncé. La moyenne des densités optiques de ces 9 plantes est évaluée à 1,022 et l'écart type $\sigma^{2}=0,624$. Ce résultat rappelle beaucoup celui enregistré avec le donneur originel $\mathrm{H} 3059-7$.

La plante $A 4$ rouge ( $D O=1,378$ ) ne donne pas de bicolore typique. La valeur moyenne des DO des $19 \mathrm{EmII}$ issus de A4 est estimée à 1,385, donc égale à celle du donneur; cependant la variation autour de cette moyenne reste élevée : $\sigma^{2}$ $=0,589$.

\section{DISCUSSION}

Les disjonctions et les nets effets d'inbreeding observés chez les descendances autofécondées et consanguines de $\mathrm{A} 15$ prouvent que cette plante ne provient pas de pollen réduit.

L'hypothèse selon laquelle, certaines plantes Em proviendraient de noyaux spermatiques non réduits pourrait alors être formulée; la renoncule présente en effet des grains de pollen de taille très hétérogène.

\section{Cependant :}

- les anthères qui engendrent des embryons Em sont généralement indéhiscentes, aucun pollen n'est visible dans leur voisinage ou à leur sur- face; par contre, lorsque par écrasement, s'échappe du pollen qui entoure l'anthère nous n'avons jamais observé d'embryons (peut-être le stade de prélèvement est-il alors trop tardif).

- les plantes $C$ provenant de l'intérieur de l'anthère sont presque toujours très peu vigoureuses et stériles, plusieurs sont haploïdes $(n=$ 8) et donc issues obligatoirement du pollen réduit. L'absence totale d'haploïdes ou d'haploïdes doublés parmi les plantes provenant de la surface de l'anthère exclut une origine pollinique de ces plantes Em.

- Les noyaux spermatiques non réduits devraient faire apparaître des structures homozygotes SxSx, SySy et en conséquence des combinaisons consanguines intercompatibles ( $\mathrm{Sx} x \mathrm{x}$ $x$ SySy; SxSx x SxSy...) qui n'ont jamais été observées.

Compte tenu de tous ces faits, seule l'origine somatique des plantes Em est vraisemblable et la variation relatée est d'origine somaclonale.

Les caractères nouveaux sont stables et se transmettent sexuellement par la voie mâle, ils sont donc probablement portés par l'ADN nucléaire. C'est le cas en particulier du système d'auto-incompatibilité. Plusieurs auteurs ont déjà relaté des mutations d'allèles $S$ après culture in vitro d'anthères : Cappadocia et al (1986) chez Solanum chacoense, Sree Ramulu (1982) chez Lycopersicon peruvianum. Dans nos essais le comportement de la plante diploïde A15 et de ses descendants s'interprète bien par une mutation d'un allèle $S$ en une forme d'autofertilité.

Sree Ramulu (1982) remarque également que la fréquence des altérations au locus $S$ est très élevée chez les plantes provenant d'anthères, nulle chez les plantes issues d'entrenceuds de tiges. Une telle incidence de l'organe d'origine sur l'importance de la variation somaclonale est soulignée par d'autres auteurs; ainsi Khalid et al (1989) montrent que chez Chrysanthemum morifolium les pétales régénèrent des plantes beaucoup plus diversifiées que les feuilles. Dans le cas de la renoncule, les plantes régénérées à partir de tronçons de thalamus sont conformes à leur donneur (et en particulier S7002-6) (Meynet et Duclos, 1990a), au contraire les plantes provenant d'anthères par embryogenèse somatique révèlent une très grande variation.

L'importance considérable de la variation décrite chez cette espèce suggère l'existence d'un phénomène de transposition. Contrairement aux exemples fameux d'éléments transposables affectant un gène de synthèse anthocyanique et 
se manifestant spontanément par le phénotype de bigarrure (Nevers et al, 1986), la renoncule posséderait des éléments transposables qui seraient activés dans les tissus somatiques de l'anthère (épiderme) peut-être sous l'influence de la culture in vitro ou du traitement à la chaleur, mais qui resteraient inactifs dans le thalamus. De plus, il existerait une relation entre la présence d'un élément transposable susceptible d'affecter l'intensité et la répartition pigmentaire et le caractère bicolore du génotype donneur.

Nous devons remarquer enfin que seul le donneur hétérozygote $57002-6$ a fait apparaître des plantes dérivées acyaniques ou à cyanidine correspondant à une perte fonctionnelle des allèles dominants $A$ et $D p$. Cette observation suggère une relation probable entre la production de variants et l'état hétérozygote du matériel de départ.

\section{CONCLUSION}

La culture in vitro d'anthère révèle chez la renoncule une variation somaclonale extrêmement spectaculaire. Bien que son déterminisme ne soit pas connu, le phénomène est très intéressant pour le sélectionneur :

- les caractères nouveaux apparus sont stables et héritables, les variants peuvent donc être exploités immédiatement, soit comme cultivar, soit comme géniteur.

- la variation ne semble pas aléatoire; par exemple seules les plantes bicolores ont donné une très grande variation de coloris. L'extrapolation de cette observation à l'ensemble de l'espèce et à d'autres espèces ornementales qui présentent le même phénotype bicolore serait d'un intérêt évident.

\section{REMERCIEMENTS}

Nous remercions $E$ Berthelé pour son aide technique et $F$ Pécate qui a participé à ce travail dans le cadre d'un stage de fin d'étude de l'Ecole Nationale Supérieure Agronomique de Toulouse.

\section{RÉFÉRENCES}

Cappadocia M, Cheng DSK, Ludlum-Simonette R (1986) Self-compatibility in doubled haploids and their $\mathrm{F} 1$ hybrids, regenerated via anther culture in self-incompatible Solanum chacoense Bitt Theor App/ Genet 72, 66-69

Khalid N, Davey MR, Power JB (1989) An assessment of somaclonal variation in Chrysanthemum morifolium: the generation of plants of potential commercial value. Sci Hortic 38, 287-294

Meynet J (1974) Travaux d'amélioration de la renoncule. Eucarpia, Fréjus 5-6 mars 1974, 4-17

Meynet J, Duclos A (1990a) Culture in vitro de la renoncule des fleuristes (Ranunculus asiaticus $\mathrm{L}$ ) I. Néoformation et multiplication végétative in vitro de plantes à partir de tronçons de thalamus. Agronomie 10, 157-162

Meynet J, Duclos A (1990b) Culture in vitro de la renoncule des fleuristes (Ranunculus asiaticus $L$ ) II. Production de plantes par culture in vitro d'anthères. Agronomie 10

Nevers P, Shepherd NS, Saedler H (1986) Plant transposable elements. Adv Bot Res 12, 103-203

Sree Ramulu K (1982) Genetic instability at the S locus of $L y c o p e r s i c o n$ peruvianum plants regenerated from in vitro culture of anthers : Generation of new $\mathrm{S}$ specificities and $\mathrm{S}$ allele reversions. Heredity 49 , 319-330 